La agresividad en la infancia: el estilo de crianza parental como factor relacionado European Journal of Education and Psychology, vol. 2, núm. 3, noviembre, 2009, pp. 211-222,

\title{
Editorial CENFINT
}

\section{España}

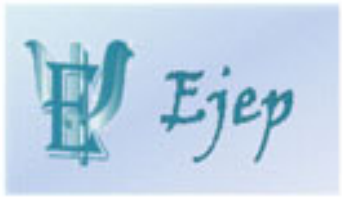

\author{
European Journal of Education and Psychology, \\ ISSN (Versión impresa): 1888-8992 \\ ejep@ejep.es \\ Editorial CENFINT \\ España
}

¿Cómo citar? | Fascículo completo | Más información del artículo I Página de la revista

\section{www.redalyc.org}

Proyecto académico sin fines de lucro, desarrollado bajo la iniciativa de acceso abierto 


\title{
La agresividad en la infancia: el estilo de crianza parental como factor relacionado
}

\author{
Antonio F. Raya, Ma José Pino y Javier Herruzo \\ Universidad de Córdoba (España)
}

\begin{abstract}
El presente estudio se propone analizar la posible relación existente entre la agresividad en los niños medida por sus padres a través del BASC (Sistema de Evaluación de la Conducta de Niños y Adolescentes) y el estilo de crianza según el PCRI (Cuestionario de Crianza Parental) compuesto por siete factores: apoyo, satisfacción con la crianza, compromiso, comunicación, disciplina, autonomía y distribución de rol, en una muestra de 338 niños (182 niños y 156 niñas) entre 3 y 14 años. Los resultados muestran la existencia de una relación significativa entre la agresividad en los niños y la mayoría de los factores del estilo de crianza parental. Además se establece un modelo capaz de predecir el $27 \%$ de la varianza con respecto a la agresividad en los niños, compuesto por la disciplina de ambos progenitores, el compromiso y la satisfacción con la crianza de los padres y la autonomía de las madres. Finalmente se discute la utilidad de estos resultados para el planteamiento de estrategias de intervención en el ámbito familiar basadas en el estilo disciplinario.
\end{abstract}

Palabras clave: Agresividad, padres, estilo parental, disciplina.

Aggression in childhood: Parenting style as related factor. This current study proposes to analyse the possible relationship which exists between aggression in children reported by parents through the BASC (Behaviour Assessment System for Children), and the parenting style according to the PCRI (Parent-Child Relationship Inventory) composed of seven factors such as support, satisfaction with parenting, involvement, communication, limit setting, autonomy granting, and role orientation, in a sample of 338 children (182 male \& 156 female) between 3 and 14 years old. The results show the existence of a significant relationship between aggression in children and the majority of the parenting factors. Furthermore, a model is established which is able to predict $27 \%$ of the variance with respect to aggression in children, made up by limit setting from both parents, involvement and satisfaction with parenting from the fathers, and autonomy granting from the mothers. Finally, the utility of results to raise intervention strategies within the family based on limit setting is discussed.

Key words: Aggression, parents, parenting style, limit setting.

Correspondencia: Antonio Félix Raya Trenas. Departamento de Psicología. Facultad de Ciencias de la Educación. Universidad de Córdoba. C/San Alberto Magno s/n. 14071 Córdoba. E-mail: m02ratra@uco.es 
Si bien la agresividad puede ser entendida como un patrón adaptativo desde el punto de vista etológico, las agresiones son un problema de suma importancia para la humanidad en general y para las sociedades occidentales en particular. Las altas tasas de fenómenos como la violencia de género, violencia intrafamiliar, malos tratos en la infancia, delincuencia, etc., sitúan a este fenómeno como un componente importante de una variada gama de problemáticas psicosociales que justifican sobradamente su estudio desde un punto de vista psicológico. Dentro de este conjunto de problemas, merece una especial atención por su elevada prevalencia el maltrato entre iguales o bullying, pudiendo señalar a las agresiones injustificadas en la primera infancia como un claro precursor del mismo en los años posteriores (Monks, Ortega y Torrado, 2002; Ortega y Monks, 2005; Romera, Ortega y Monks, 2008).

Los numerosos intentos realizados para definir lo que entendemos por agresión o conducta agresiva, demuestran que no se trata de un término unívoco, sino que presenta distintas connotaciones, tanto psicológicas como sociales, morales, etc. Entre estos intentos definitorios se puede destacar el realizado por Bandura (1973), que la definió como una conducta adquirida controlada por reforzadores, que es perjudicial y destructiva, o la definición elaborada posteriormente por Edmunds y Kendrick (1980), que definieron el término agresividad instrumental como disposición a manifestar conductas nocivas como medio de obtener refuerzos del exterior. Ambas definiciones hacen referencia a la interacción del sujeto con el entorno, que se concreta en comportamientos como gritos, golpes, amenazas, daños, ataques, invasión, humillación, etc. Estas expresiones conductuales de variable intensidad se pueden agrupar en verbales y no verbales, físicas y psicológicas, tienen un fin y una intencionalidad clara en el daño producido (Fariz et al., 2002). En el Behavior Assessment System for Children (BASC), Reynolds y Kamphaus (2004) también inciden en la multidimensionalidad del fenómeno y definen la agresión como tendencia a hacer daño físico o emocional a otros, abarcando tanto las agresiones físicas, con conductas como romper las propiedades de otros, golpear a otros o hacer daño a los animales, como las agresiones verbales, manifestadas mediante conductas como discutir, criticar, amenazar, insultar o culpar a otros. Existe una alta correlación entre este constructo y el de conducta agresiva contenida en el Child Behavior Check-List (CBCL) de Achenbach (1991). Por otro lado, tanto en el DSM-IV-TR como en versiones anteriores, la conducta inapropiadamente agresiva es incluida dentro de los trastornos de tipo externalizante y muestra una alta correlación con otros trastornos del comportamiento como el TDAH o el Trastorno Oposicionista Desafiante (APA, 2002).

En una extensa revisión de las distintas teorías psicológicas sobre la agresión, Carrasco y González (2006) mencionan que ya desde los años 70 e incluso antes se han ido desarrollando numerosas teorías que relacionan la conducta agresiva en la infancia con los hábitos de crianza de los padres. Entre las variables más importantes que han 
sido estudiadas figuran el rechazo, la falta de apoyo y afecto, el uso del castigo para controlar la conducta del niño, la falta de supervisión y comunicación y una disciplina inconsistente. Por otro lado, numerosos estudios llevados a cabo en los últimos años, también con población española, como el de Roa y Del Barrio (2002) o Tur, Mestre y Del Barrio (2004a, 2004b) han demostrado que un estilo educativo excesivamente autoritario o por el contrario excesivamente permisivo favorece la aparición de conductas agresivas en el niño, mientras que un estilo autoritativo, caracterizado por altos niveles de apoyo, supervisión y flexibilidad actúa como factor protector de la conducta agresiva. También existen otros elementos familiares relacionados con la tendencia a la agresión como pueden ser la violencia paterna, el alcoholismo, la depresión, la calidad de las relaciones y las desavenencias matrimoniales, el divorcio o la monoparentalidad (Del Barrio y Roa, 2006).

Partiendo del modelo planteado por Darling y Steinberg (1993) según el cual los padres muestran un estilo educativo más o menos estable que se manifiesta mediante unas prácticas de crianza específicas, numerosos estudios han tratado de relacionar la conducta agresiva no sólo con los distintos estilos de crianza, sino también con otros constructos más concretos referentes a la interacción diaria con los hijos. Uno de estos constructos es el establecimiento de límites (limit setting), también denominado disciplina en algunas ocasiones, que hace referencia a la consistencia de las normas establecidas por los padres y la inducción razonada hacia su cumplimiento por parte de los hijos. Diversos estudios han encontrado en esta variable un factor protector importante ante la conducta agresiva (Côté, Vaillancourt, LeBlanc, Nagin y Tremblay, 2006; Romano, Tremblay, Boulerice y Swisher, 2005). Por el contrario, los estudios que han medido la inconsistencia en el estilo normativo han encontrado una relación directa entre esta variable y la conducta agresiva (Mazefsky y Farrell, 2005; McCoy, Frick, Loney y Ellis, 1999; Vaillancourt, Miller, Fagbemi, Côté y Tremblay, 2007). En otras ocasiones, se han comparado distintos tipos de disciplina, observándose que una disciplina agresiva, caracterizada por un bajo nivel de razonamiento y altos niveles de agresividad tanto física como verbal y emocional, genera conductas agresivas en los propios hijos mientras que una disciplina menos agresiva y basada en el razonamiento no lo hace (Sheehan y Watson, 2008).

Por otro lado, estudios que han contemplado aspectos como la falta de supervisión y compromiso con la crianza, la sitúan, junto con unas pautas disciplinarias inconsistentes, como un factor determinante de la conducta agresiva en los hijos (p. e. Knutson, DeGarmo y Reid, 2004; Mazefsky y Farell, 2005; McCoy et al., 1999). Como ejemplo de esta relación, en el estudio llevado a cabo por Mazefsky y Farell (2005) con 1196 alumnos de Educación Secundaria pertenecientes a zonas rurales de Estados Unidos, se concluyó que un estilo parental caracterizado por bajos niveles de supervisión y disciplina junto con un bajo nivel de apoyo parental favorecían la conducta 
agresiva en los chicos, sobre todo cuando estos presenciaban violencia en su entorno y provocación por parte de otros. Entre la falta de supervisión y la autonomía existe una línea bastante delgada, de modo que algunos estudios llevados a cabo por Mestre y sus colaboradoras le dan a esta última un significado positivo en la medida en que fomenta la responsabilidad y la madurez, actuando como factor protector ante la conducta agresiva (Mestre, Samper, Tur y Díez, 2001; Mestre, Frías, Samper y Nácher, 2003), mientras que en otros estudios llevados a cabo posteriormente por las mismas autoras plantean que la autonomía puede resultar perniciosa cuando se encuadra dentro de un estilo parental negligente (Mestre, Samper, Nácher, Cortés y Tur, 2004; Samper, Aparici y Mestre, 2006).

Estudios anteriores llevados a cabo con población española han mostrado que variables como la autonomía o la disciplina, pertenecientes al estilo de crianza de padres y madres, guardan una estrecha relación con determinados problemas de comportamiento en los hijos como la hiperactividad y la impulsividad (Raya, Herruzo y Pino, 2008) o como la propia conducta agresiva (Mestre et al., 2004; Samper et $a l$, 2006). En definitiva, esta relación entre determinados aspectos del estilo de crianza y ciertas manifestaciones conductuales inadecuadas como la agresión, nos permite plantear que, llevando a cabo una medición de determinadas características del estilo de crianza de los padres, se podría predecir en cierta medida la presencia de otros problemas de adaptación en los hijos como es el caso de la conducta agresiva. En este sentido, este estudio se propone describir la relación existente entre la conducta agresiva en una muestra de escolares informada por los padres por medio del BASC y el estilo de crianza de sus padres y madres según el PCRI. Además, se pretende elaborar un modelo predictor de la conducta agresiva en los hijos compuesto por distintos factores del estilo de crianza de los padres y madres.

\section{METODO}

\section{Participantes}

Para la selección de un amplio número de participantes de estatus socioeconómico medio, se recurrió a tres centros de Educación Infantil, Primaria y Secundaria, situados en dos localidades de la provincia de Córdoba y una de la provincia de Jaén. Así, tras suministrar un total de 500 cuestionarios, se obtuvo información de 338 chicos y chicas con sus respectivos padres y madres.

La edad media de los padres era de 40.15 (DT= 5.573), con un rango entre 27 y 70 años, mientras que la edad media de las madres era de 38.22 (DT= 5.097), situándose el rango en este caso entre 25 y 56 años.

Por su parte, la muestra de hijos estaba compuesta por 182 niños y 156 niñas, con una edad media de 8.36 (DT= 3.123), con un mínimo de 3 años, un máximo de 14 . 


\section{Instrumentos}

Para la recogida de la información se han empleado los siguientes instrumentos:

- Sistema de evaluación de la conducta de niños y adolescentes (BASC) (Reynold y Kamphaus, 2004): es una adaptación al castellano del original en lengua inglesa Behavior Assessment System for Children.

Evalúa una amplia gama de dimensiones patológicas y adaptativas, mediante distintas fuentes de información y distintos métodos. Para este estudio se ha usado el cuestionario para padres.

Este cuestionario se divide en tres niveles según la edad (3-6, 6-12, 12-18) y presenta un índice de consistencia interna entre .70 y .90 , según las escalas. Respecto a la correlación test-retest, los valores son .85, .88 y .70 para los tres niveles del cuestionario.

De las distintas escalas que contempla el instrumento, para este estudio se ha usado la escala denominada "Agresividad", definida en el propio instrumento como tendencia a actuar de forma hostil que amenaza a otros. Esta escala presenta unos índices de consistencia interna entre .79 y .75, dependiendo de las edades de los sujetos.

Las puntuaciones obtenidas en cualquiera de las escalas son transformadas en puntuaciones $\mathrm{T}$, que señalan la distancia de una puntuación con respecto a la media del grupo normativo y permiten establecer comparaciones entre sujetos de diferentes edades. Estas puntuaciones $\mathrm{T}$ pueden oscilar entre 0 y 100 y presentan una media de 50 y una D.T. de 10. En base a estas puntuaciones T se establecen distintos niveles, de modo que puntuaciones por debajo de 30 se consideran muy bajas, por debajo de 40 bajas, entre 40 y 60 nivel medio o normal, por encima de 60 en riesgo y por encima de 70 clínicamente significativo.

- Cuestionario de Crianza Parental (PCRI-M) de Roa y Del Barrio (2001) adaptado del Parent-Child Relationship Inventory (Gerard, 1994): este cuestionario mide las actitudes paternas y maternas hacia la crianza de sus hijos.

Está compuesto por 78 ítems con cuatro opciones de respuesta, que se agrupan en siete escalas:

Apoyo: nivel de apoyo social y emocional que una madre o padre está recibiendo.

Satisfacción con la crianza: satisfacción que obtiene una madre o padre por el hecho de serlo.

Compromiso: grado de interacción y conocimiento que los padres tienen de su hijo.

Comunicación: percepción que los padres tiene de la efectividad de la comunicación con su hijo. 
Disciplina: nivel de exigencia de cumplimiento de las normas impuesto por los padres.

Autonomía: habilidad de los padres para proporcionar independencia al hijo.

Distribución de rol: actitudes de los padres acerca del papel que desempeña el género en la crianza.

En general, una alta puntuación en cualquiera de las escalas indica una buena actitud hacia la crianza (estilo democrático), mientras que puntuaciones bajas son signo de un estilo de crianza poco efectivo.

Este instrumento presenta una consistencia interna obtenida mediante el coeficiente alfa de Cronbach, que oscila entre 0.48 de la escala de Autonomía y 0.68 de la escala de Compromiso. Además, según Roa y Del Barrio, presenta una buena validez de constructo, dadas las altas correlaciones entre las distintas escalas del cuestionario, sobre todo en las escalas más importantes de la crianza como son CompromisoSatisfacción con la crianza (0.58), Compromiso-Comunicación (0.64), Disciplina-Apoyo (0.52), Disciplina-Autonomía (0.44) y Satisfacción con la Crianza-Disciplina (0.43).

\section{Procedimiento}

Para contactar con las familias participantes recurrimos a los centros donde estaban escolarizados sus hijos. El primer paso fue obtener permiso de los equipos directivos y consejos escolares de los distintos centros para participar en el estudio. Para informar a las familias por escrito y hacerles llegar el instrumento contamos con la colaboración de los tutores de los distintos cursos, que se encargaron tanto de distribuir como de recoger de los cuestionarios. Las familias que accedieron a colaborar de manera voluntaria cumplimentaron el BASC en su forma P (padres) y en sus distintas versiones según la edad de los chicos y el PCRI tanto por parte del padre como de la madre.

Tras recoger los cuestionarios se pasó a la tabulación de los datos, transformación de las puntuaciones del BASC en puntuaciones T como se indica en la descripción del instrumento y realización de los análisis con el paquete estadístico SPSS.

El estudio se ha realizado con un diseño ex post facto prospectivo en el que se ha realizado un análisis de regresión múltiple para obtener los factores predictores de la agresividad. Por lo tanto, las variables empleadas en el estudio fueron las siguientes: como variable dependiente (VD) se ha usado la puntuación $\mathrm{T}$ en agresividad del hijo valorada por sus padres, y como variables predictoras los factores del estilo de crianza tanto del padre como de la madre medidos con el PCRI-M, que son: apoyo, satisfacción con la crianza, compromiso, comunicación, disciplina, autonomía y distribución de rol.

Previamente al análisis de regresión múltiple se han descrito las puntuaciones obtenidas en los distintos instrumentos y se ha calculado el coeficiente de correlación de Pearson entre la agresividad y los factores del estilo de crianza de padres y madres. 


\section{RESULTADOS}

Para la variable agresividad, se ha obtenido una puntuación T media de 48.33 $(\mathrm{DT}=9.974)$, que resultó ser un poco más baja que la media establecida por el instrumento $(\mathrm{T}=50)$. Las puntuaciones obtenidas por los participantes se sitúan en un rango entre 29 y 90.

Respecto a la clasificación de los sujetos en función de los niveles establecidos por el BASC, la figura 1 muestra el porcentaje de sujetos que han sido clasificados como muy adaptados $(\mathrm{T}<30)$, adaptados $(30<\mathrm{T}<40)$, dentro de un rango normal $(40<\mathrm{T}<60)$, en riesgo $(\mathrm{T}>60)$ y clínicamente significativos $(\mathrm{T}>70)$.

Figura 1. Clasificación de los chicos según su puntuación en agresividad

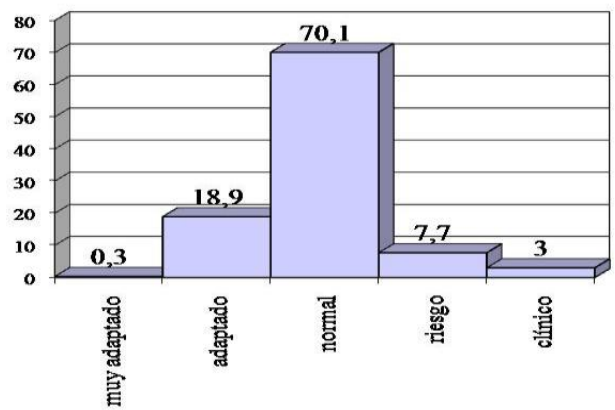

口agnesividad

Posteriormente, para identificar aquellas variables relacionadas con la agresividad, se calculó el coeficiente de correlación de Pearson entre la agresividad y todos los factores del PCRI de padres y madres, cuyas puntuaciones medias se muestran en la tabla 1. Todas las variables han correlacionado de manera inversa con la agresividad. Además, el apoyo, la satisfacción con la crianza, el compromiso, la comunicación y la disciplina de ambos progenitores lo han hecho de manera significativa, luego altas puntuaciones en estas variables están relacionadas con bajas puntuaciones en agresividad.

Tabla 1. Puntuación media para cada factor del PCRI y coeficientes de correlación de Pearson entre los factores del PCRI de padres y madres y la agresividad

\begin{tabular}{|c|c|c|c|c|c|c|c|}
\hline PCRI padre & Media & DT & $\begin{array}{c}\text { Correlación con } \\
\text { Agresividad }\end{array}$ & PCRI madre & Media & DT & $\begin{array}{c}\text { Correlación con } \\
\text { Agresividad }\end{array}$ \\
\hline Apoyo & 25.28 & 3.630 & $-0.214 *$ & Apoyo & 23.77 & 4.129 & $-0.297 * *$ \\
\hline Satisfacción & 34.75 & 4.070 & $-0.277 * *$ & Satisfacción & 34.82 & 4.255 & $-0.226 * *$ \\
\hline Compromiso & 43.67 & 4.418 & $-0.280^{* *}$ & Compromiso & 45.02 & 3.965 & $-0.279 * *$ \\
\hline Comunicación & 28.62 & 3.360 & $-0.172 * *$ & Comunicación & 29.80 & 3.526 & $-0.125^{*}$ \\
\hline Disciplina & 32.03 & 4.498 & $-0.372 * *$ & Disciplina & 31.81 & 4.876 & $-0.436^{* * *}$ \\
\hline Autonomía & 24.84 & 3.069 & -0.109 & Autonomía & 25.32 & 3.513 & -0.085 \\
\hline Distrib. rol & 27.55 & 4.137 & -0.106 & Distrib. rol & 28.52 & 4.029 & -0.089 \\
\hline
\end{tabular}


Finalmente, para estudiar el carácter predictivo de los factores del PCRI respecto a la agresividad, se aplicó el análisis de regresión múltiple mediante el que se pretende obtener un modelo capaz de predecir la posibilidad de presentar una alta puntuación en agresividad. Las cinco variables que componían el modelo con mayor capacidad predictora eran la disciplina de la madre, la disciplina del padre, el compromiso del padre, la autonomía de la madre y la satisfacción con la crianza del padre, como muestra la tabla 2.

En el quinto paso se obtuvo una $\mathrm{R}^{2}$ de 0.270 , por lo que se puede decir que las variables disciplina de la madre, disciplina del padre, compromiso del padre, autonomía de la madre y satisfacción con la crianza del padre predicen el $27 \%$ de la variabilidad respecto a la agresividad. Para todas estas variables se han obtenido coeficientes Beta negativos excepto para la autonomía de la madre, por lo que esta variable es predictiva de una puntuación alta en agresividad cuando presenta puntuaciones también altas. Para el resto de las variables ocurre lo contrario.

Todas las variables incluidas hacen una aportación interesante al modelo, que mejora ostensiblemente en cada paso. Por su parte, los estadísticos de colinealidad que nos informan sobre la independencia de cada factor respecto a los demás, ofrecen unos resultados bastante buenos, teniendo en cuenta que el valor de tolerancia está más próximo a 1 que a 0 y que el factor de inflación de la varianza (FIV) no se aleja demasiado de 1.

\begin{tabular}{|c|c|c|c|c|c|c|c|c|c|c|c|c|}
\hline \multirow[t]{2}{*}{ Variable } & \multirow[t]{2}{*}{$\mathrm{R}$} & \multirow[t]{2}{*}{$\mathrm{R}^{2}$} & \multirow[t]{2}{*}{$\mathrm{R}^{2}$ corr. } & \multirow[t]{2}{*}{$\mathrm{F}$} & \multirow[t]{2}{*}{ sig. } & \multirow[t]{2}{*}{ B } & \multirow[t]{2}{*}{$\mathrm{t}$} & \multirow[t]{2}{*}{ sig. } & \multicolumn{2}{|c|}{ Int. para B 95\% } & \multicolumn{2}{|c|}{ Colinealidad } \\
\hline & & & & & & & & & Inf. & Sup. & Tol. & FIV \\
\hline Paso 1 & 0.424 & 0.180 & 0.177 & 60.61 & .000 & & & & & & & \\
\hline Constante & & & & & & 76.722 & 21.018 & .000 & 69.536 & 83.908 & & \\
\hline Disciplina madre & & & & & & -0.887 & -7.785 & .000 & -1.111 & -.663 & 1.000 & 1.000 \\
\hline Paso 2 & 0.469 & 0.220 & 0.214 & 38.92 & .000 & & & & & & & \\
\hline Constante & & & & & & 86.139 & 19.809 & .000 & 77.579 & 94.699 & & \\
\hline Disciplina madre & & & & & & -0.672 & -5.381 & .000 & -.918 & -.426 & .794 & 1.260 \\
\hline Disciplina padre & & & & & & -0.506 & -3.784 & .000 & -.769 & -.243 & .794 & 1.260 \\
\hline Paso 3 & 0.496 & 0.246 & 0.238 & 29.95 & .000 & & & & & & & \\
\hline Constante & & & & & & 99.471 & 16.381 & .000 & 87.517 & 111.42 & & \\
\hline Disciplina madre & & & & & & -0.629 & -5.082 & .000 & -.873 & -.386 & .784 & 1.276 \\
\hline Disciplina padre & & & & & & -0.435 & -3.256 & .001 & -.698 & -.172 & .770 & 1.298 \\
\hline Compromiso padre & & & & & & -0.389 & -3.097 & .002 & -.636 & -.142 & .929 & 1.076 \\
\hline Paso 4 & 0.509 & 0.259 & 0.248 & 23.93 & .000 & & & & & & & \\
\hline Constante & & & & & & 92.651 & 13.610 & .000 & 79.249 & 106.05 & & \\
\hline Disciplina madre & & & & & & -0.779 & -5.519 & .000 & -1.057 & -.501 & .595 & 1.680 \\
\hline Disciplina padre & & & & & & -0.397 & -2.965 & .003 & -.661 & -.133 & .757 & 1.321 \\
\hline Compromiso padre & & & & & & -0.373 & -2.984 & .003 & -.618 & -.127 & .926 & 1.080 \\
\hline Autonomía madre & & & & & & 0.383 & 2.162 & .032 & .034 & .731 & .751 & 1.332 \\
\hline $\begin{array}{l}\text { Paso } 5 \\
\text { Constante }\end{array}$ & 0.519 & 0.270 & 0.256 & 20.15 & .000 & 94.947 & 13.825 & .000 & 81.426 & 108.47 & & \\
\hline Disciplina madre & & & & & & -0.792 & -5.638 & .000 & -1.069 & -.516 & .594 & 1.684 \\
\hline Disciplina padre & & & & & & -0.338 & -2.477 & .014 & -.607 & -.069 & .721 & 1.386 \\
\hline Compromiso padre & & & & & & -0.241 & -1.715 & .087 & -.518 & .036 & .722 & 1.385 \\
\hline Autonomía madre & & & & & & 0.434 & 2.440 & .015 & .084 & .784 & .735 & 1.360 \\
\hline Satisfacción padre & & & & & & -0.310 & -1.994 & .047 & -616 & -.004 & .691 & 1.447 \\
\hline
\end{tabular}




\section{DISCUSION}

Los datos descriptivos indican una puntuación media bastante cercana a la media normativa. Por su parte, la distribución en base a los criterios de clasificación del BASC presenta una alta concentración de sujetos en el rango "normal", mientras que los sujetos clínicamente significativos se reducen a un $3 \%$, lo que resulta bastante representativo de la población general, donde las tasas de prevalencia para el trastorno disocial oscilan entre el 2 y el 16\% (APA, 2002).

Este estudio se proponía analizar la relación existente entre el estilo de crianza de ambos progenitores y la conducta agresiva en los hijos. En la introducción se apuntaba cierta relación que numerosos autores habían descrito entre determinados aspectos del estilo de crianza de los padres y la conducta agresiva en los hijos, de manera que podrían llegar a establecerse determinados modelos familiares directamente relacionados con la misma. En este sentido, los resultados obtenidos reflejan una elevada relación entre determinadas características del estilo de crianza y la conducta agresiva, de manera que una combinación de puntuaciones altas en autonomía de la madre junto con puntuaciones bajas en satisfacción con la crianza y compromiso del padre y disciplina de ambos progenitores, influyen significativamente en el incremento de la probabilidad de que se dé una puntuación elevada en agresividad según el BASC.

En general, la mayoría de las variables que se han incluido en el modelo, a excepción de la autonomía de la madre, actúan como predictoras de una alta probabilidad de puntuar en la zona de riesgo cuando presentan puntuaciones bajas y como predictoras de una alta probabilidad de puntuar en la zona de baja agresividad cuando las puntuaciones son altas. Esto coincide en buena medida con lo encontrado en estudios anteriores como los de Côté et al. (2006) o Romano et al. (2005) respecto a la disciplina y el establecimiento de una normas consistentes. También coincide con lo descrito por Mestre et al. (2004) o Samper et al. (2006), que señalaban una excesiva autonomía y falta de compromiso como factores de riesgo respecto a la conducta agresiva en los hijos. Una nueva variable, como es la satisfacción con la crianza del padre, ha sido incluida en el modelo obtenido en este estudio, lo que da buena cuenta de los efectos negativos de este tipo de problemas en los hijos sobre el bienestar de los padres y de la familia en general.

Los resultados obtenidos también muestran coherencia con lo obtenido por Roa y Del Barrio $(2001,2002)$ que, de acuerdo con los modelos de crianza propuestos por Baumrind (1991), identificaban puntuaciones elevadas en las principales escalas del PCRI con una buena crianza, lo que estaría en línea con el modelo autoritativo. Al mismo tiempo, ambas autoras afirmaban que las puntuaciones bajas denotaban 
problemas para llevar a cabo la crianza de una forma adecuada, identificándose así con el modelo autoritario.

En esta línea, teniendo en cuenta que la falta de disciplina era una de las principales características con que se identifica al modelo permisivo y que, a su vez, este factor ha resultado decisivo en la predicción de la conducta agresiva, coincidimos con la mayoría de los autores al señalar al modelo autoritativo como predictor de una baja puntuación en agresión.

A pesar de haber obtenido la información por medio de autoinformes de los padres y madres, el elevado número de sujetos analizados proporciona una información muy valiosa para el planteamiento de pautas de prevención e intervención con familias de niños que manifiestan una elevada tasa de conductas agresivas, pues puede orientar sobre algunos aspectos concretos de la práctica educativa diaria que pueden ser modificados, como establecer unas normas claras y asegurar su cumplimiento, supervisar las distintas tareas de los hijos e implicar a todos los miembros de la unidad familiar en las distintas tareas de crianza para favorecer la compatibilidad con el desempeño laboral de ambos progenitores.

Por último, de cara al futuro, se podrían llevar a cabo nuevos estudios que aporten información sobre algunos aspectos aún por aclarar como las posibles diferencias en las formas de disciplina empleadas por ambos progenitores. Además, el presente estudio abre una amplia vía de trabajo, en la que se podrían tratar otros problemas tanto de tipo externalizante como internalizante.

\section{REFERENCIAS}

Achenbach, T.M. (1991). Child Behavior Check-List. Burlington, VT: Author.

American Psychiatric Association (2002). Manual diagnóstico y estadístico de los trastornos mentales, $4^{\text {a }}$ edición, texto revisado (DSM-IV-TR). Barcelona: Masson (Edición original, 2000).

Bandura, A. (1973). Aggression: a social learning analysis. Englewood Cliffs, NJ: Prentice Hall.

Baumrind, D. (1991). Parenting styles and adolescent development. En J. Brooks-Gun, R. Lerner \& A.C. Petersen (Eds.), The encyclopaedia of adolescence (pp. 746-758). New York: Garland.

Carrasco, M.A. y González, M.J. (2006). Aspectos conceptuales de la agresión: definición y modelos explicativos. Acción Psicológica, 2(4), 7-38.

Côté, S.M., Vaillancourt, T., LeBlanc, J.C., Nagin, D.S. \& Tremblay, R.E. (2006). The Development of Physical Aggression from Toddlerhood to Pre-Adolescence: A Nation Wide Longitudinal Study of Canadian Children. Journal of Abnormal Child Psychology, 34(1), 71-85.

Darling, N. \& Steinberg, L. (1993). Parenting Style as Context: An Integrative Model. Psychological Bulletin, 113(3), 487-496.

Del Barrio, M.V. y Roa, M.L. (2006). Factores de riesgo y de protección en agresión infantil. Acción Psicológica, 2(4), 39-65. 
Edmunds, G. \& Kendrick, D.C. (1980). The measurement of human aggressiveness. Chichester: Ellis Horwood.

Fariz, M.D., Mías, C.D. y Borges de Moura, C. (2002). Comportamiento agresivo y terapia cognitivo-comportamental en la infancia. En V.E. Caballo y M.A. Simón (Coords.), Manual de psicopatología clínica infantil y del adolescente. Trastornos específicos (pp. 57-75). Madrid: Pirámide.

Gerard, A. (1994). Parent-Child Relationship Inventory: Manual. Los Angeles: Western Psychological Services.

Knutson, J.F., DeGarmo, D.S. \& Reid, J.B. (2004). Social Disadvantage and Neglectful Parenting as Precursors to the Development of Antisocial and Aggressive Child Behavior: Testing a Theoretical Model. Aggressive Behavior, 30, 187-205.

Mazefsky, C.A. \& Farell, A.D. (2005). The Role of Witnessing Violence, Peer Provocation, Family Support, and Parenting Practices in the Aggressive Behavior of Rural Adolescents. Journal of Child and Family Studies, 14(1), 71-85.

McCoy, M.G., Frick, P.J., Loney, B.R. \& Ellis, M.L. (1999). The Potential Mediating Role of Parenting Practices in the Development of Conduct Problems in a Clinic-Referred Sample. Journal of Child and Family Studies, 8(4), 477-494.

Mestre, V., Frías, M.D., Samper, P. y Nácher, M.J. (2003). Estilos de crianza y variables personales como factores de riesgo de la conducta agresiva. Revista Mexicana de Psicología, 20(2), 189-199.

Mestre, V., Samper, P., Nácher, M.J., Cortés, M. y Tur, A. (2004, septiembre). Estilos de crianza y agresividad en la infancia. Trabajo presentado en el II Congreso Hispano-Portugués de Psicología. Lisboa, Portugal.

Mestre, V., Samper, P., Tur, A. y Díez, I. (2001). Estilos de crianza y desarrollo psicosocial de los hijos. Revista de Psicología General y Aplicada, 54(4), 691-703.

Monks, C., Ortega, R. \& Torrado, E. (2002). Unjustified Aggression in preschool. Aggressive Behavior, 28, 458-476.

Ortega, R. y Monks, C. (2005). Agresividad injustificada entre preescolares. Psicothema, 17(3), 453-458.

Raya, A.F., Herruzo, J. y Pino, M.J. (2008). El estilo de crianza parental y su relación con la hiperactividad. Psicothema, 20(4), 691-696.

Reynolds, C.R. y Kamphaus, R.W. (2004). Sistema de Evaluación de la Conducta de Niños y Adolescentes. Madrid: TEA Ediciones (original edition, 1992).

Roa, L. y Del Barrio, M.V. (2001). Adaptación del cuestionario de crianza parental (PCRI-M) a población española. Revista Latinoamericana de Psicología, 33(3), 329-341.

Roa, L. y Del Barrio, M.V. (2002). Cuestionario de percepción de crianza para niños y adolescentes. Psicología Educativa, 8(1), 37-51.

Romano, E., Tremblay, R.E., Boulerice, B. \& Swisher, R. (2005). Multievel Correlates of Childhood Physical Aggression and Prosocial Behavior. Journal of Abnormal Child Psychology, 33(5), 565-578.

Romera, E.M., Ortega, R. y Monks, C. (2008). Impacto de la actividad lúdica en el desarrollo de la competencia social. International Journal of Psychology and Psychological Therapy, 8(2), 193-202.

Samper, P., Aparici, G. y Mestre, V. (2006). La agresividad auto y heteroevaluada: variables implicadas. Acción Psicológica, 2(4), 155-168.

Sheehan, M.J. \& Watson, M.W. (2008). Reciprocal Influences Between Maternal Discipline Techniques and Aggression in Children and Adolescents. Aggressive Behavior, 34, $245-$ 255. 
Tur, A., Mestre, V. y Del Barrio, M.V. (2004a). Factores moduladores de la conducta agresiva y prosocial, el efecto de los hábitos de crianza. Ansiedad y Estrés, 10, 75-88.

Tur, A., Mestre, V. y Del Barrio, M.V. (2004b). Los problemas de conducta exteriorizados e interiorizados en la adolescencia: relaciones con los hábitos de crianza y con el temperamento. Acción Psicológica, 36(3), 207-221.

Vaillancourt, T., Miller, J.L., Fagbemi, J., Côté, S. \& Tremblay, R.E. (2007). Trajectories and Predictors of Indirect Aggression: Results From a National Representative Longitudinal Study of Canadian Children aged 2-10. Aggressive Behavior 33, 314-326.

Recibido: 28 de febrero de 2009

Recepción Modificaciones: 17 de mayo de 2009

Aceptado: 20 de junio de 2009 